\title{
Anthracnose in U.S. Tea: Pathogen Characterization and Susceptibility Among Six Tea Accessions
}

\author{
James M. Orrock, ${ }^{1}$ Bala Rathinasabapathi, ${ }^{2}$ and Brantlee Spakes Richter ${ }^{1, \dagger}$ \\ ${ }^{1}$ University of Florida, Department of Plant Pathology, Gainesville, FL \\ ${ }^{2}$ University of Florida, Horticultural Sciences Department, Gainesville, FL
}

\begin{abstract}
Tea (Camellia sinensis [L.] O. Kuntze) is under investigation as a specialty crop in the United States. Anthracnose is a serious disease in global tea production, but there is no literature on the susceptibility of U.S. planting materials to this disease. We isolated a Colletotrichum species from symptomatic plants in a field trial and identified the pathogen as Colletotrichum camelliae based on morphology and sequencing of the ITS, GS, GAPDH, TUB2, and ApMat domains. A phylogenetic analysis showed that local field isolates were genetically similar to one another and grouped with isolates from $C$. sinensis in China, whereas a local isolate from an ornamental camellia $(C$. japonica) was more closely related to $C$. camelliae isolated from other Camellia spp. Six commercially

available tea accessions were evaluated in detached leaf assays for susceptibility to this anthracnose pathogen. All accessions were susceptible to infection, with Fairhope and Small Leaf having the largest lesion sizes. In field observations, Fairhope, Big Leaf, and Small Leaf consistently had lower disease severity than Georgian over two growing seasons. This work documents the impact of anthracnose on U.S. tea varieties and may help shape future directions of tea research, breeding, and recommendations for growers in establishing a novel industry.

Keywords: Camellia sinensis, Colletotrichum camelliae, Glomerella, anthracnose, leaf bioassay, tea, Florida
\end{abstract}

Tea (Camellia sinensis [L.] O. Kuntze) is currently being explored as a new specialty crop in the southern United States. Tea is the second most consumed beverage in the world (Chang 2015) and an economically important crop. Annually the United States imports approximately 126.5 metric tons of tea worth over $\$ 12.5$ billion (Tea Association of the U.S.A. 2018). Global tea production was estimated at 5,209 metric tons of tea in 2015, showing a 10-year increase of 54\% (Teeverband 2018).

Tea is a perennial crop traditionally grown in at least 66 countries (Pettigrew 2018). In the United States, the oldest and largest tea plantation is the Charleston Tea Plantation in South Carolina (originally named Pinehurst Tea Plantation), which has been operating for over 137 years on 51 ha (Pettigrew 2018; Shepard 1899). As of 2018, tea is cultivated in 17 states on 181 ha for an annual production of $700 \mathrm{~kg}$ (Pettigrew 2018). Hawaii currently has the largest number of tea growers with at least 14 growers on approximately 20 ha (Pettigrew 2018) and is also the only state with university-based extension support for tea. Florida agriculture, long dominated by citrus, is in flux due to huanglongbing disease (syn. citrus greening). Environmental conditions in north and central Florida are similar to other tea production regions in China and Taiwan (Codeminders 2017), and tea may present an alternative crop for Florida growers (Orrock et al. 2017).

Anthracnose, caused by multiple Colletotrichum spp. (teleomorph Glomerella spp.), is one of the most important economic diseases in tea worldwide (Liu et al. 2015; Shi et al. 2018; Yoshida and Takeda 2006). It has been reported in China that anthracnose of tea can result in a yield loss of 30 to 50\% (Shi et al. 2018). In Japan, where a known

${ }^{\dagger}$ Corresponding author: B. Spakes Richter; bsr@ufl.edu

Funding: Funding was provided by Florida Department of Agriculture and Consumer Services (022918).

*The $\boldsymbol{e}$-Xtra logo stands for "electronic extra" and indicates that one supplementary figure and three supplementary tables are published online.

The author(s) declare no conflict of interest.

Accepted for publication 11 November 2019.

(C) 2020 The American Phytopathological Society anthracnose-susceptible cultivar accounts for over $75 \%$ of all tea plants grown, treatment with benzimidazole fungicides has been the predominant form of disease management (Yoshida and Takeda 2006; Yoshida et al. 2010); however, Oniki et al. (1985) reported resistant strains from more than $57 \%$ of field sites surveyed in Japan.

Colletotrichum Corda is a highly complex fungal genus composed of pathogens, endophytes, epiphytes, and saprophytes. Many species of Colletotrichum will transition among these lifestyles depending on resource availability and survival strategies (Wang et al. 2016). Worldwide, at least 16 different species have been reported from tea (Chen et al. 2017; Liu et al. 2015; Shi et al. 2018; Wang et al. 2016; Yoshida and Takeda 2006). Of these, C. acutatum, C. aenigma, C. camelliae, C. endophytica, C. fructicola, C. gloeosporioides, $C$. henanense, $C$. jiangxiense, and $C$. theae-sinensis were reported to be pathogenic (Chen et al. 2017; Liu et al. 2015; Shi et al. 2018; Wang et al. 2016; Yoshida and Takeda 2006). Currently, C. camelliae has been reported in the United States on Camellia sinensis and Camellia sasanqua (Farr and Rossman 2019), C. gloeosporioides on Camellia japonica (Weir et al. 2012), and C. acutatum on many plant species including blueberry (Sreenivasaprasad and Talhinhas 2005). However, both C. acutatum and C. gloeosporioides are large species complexes that have undergone recent revisions, and it is unclear exactly which species were observed in these earlier reports (Liu et al. 2015; Wang et al. 2016; Weir et al. 2012).

C. camelliae was first reported in the United States in 1903, with specimens registered under US0397183 at the U.S. National Fungal Collections (BPI) (Farr and Rossman 2019). However, no pathogenicity testing has been conducted with the original or subsequently identified isolates, and no information is currently available about the potential impact of $C$. camelliae on commercially grown tea varieties in the United States. In 2016, we observed anthracnose-like lesions on tea accessions in a research trial at the Plant Science Research Center (PSREC) in Citra, Florida. The plants were originally obtained from nursery and greenhouse production facilities in North Carolina and Mississippi. Because similar symptoms were also observed from the same lots maintained in a greenhouse, it is likely that the pathogen originated on nursery stock.

U.S. farmers have limited plant materials available for tea production. There are currently no named cultivars available in the United States, and importation of plant materials is highly regulated. Seed 
is the only type of planting material that is relatively easy to import; therefore, many nurseries and farmers are importing uncharacterized seeds. Although the genus Camellia is well defined, distinctions among the Camellia species used to produce tea are still debated, with some defining the two major lineages as distinct species $(C$. sinensis, $C$. assamica) and others considering them to be varieties (C. sinensis var. sinensis, $C$. sinensis var. assamica) (Vijayan et al. 2009; Wei et al. 2018). The species Camellia sinensis readily outcrosses with other members of the genus Camellia, resulting in high genetic diversity (Chen and Yamaguchi 2002). Although most plants in the United States are sold under a varietal name, many were grown from seed, or vegetatively propagated from seed-grown plants, and are likely not genetically uniform. In 2018, tea plants were being sold to consumers under 24 distinct names (Supplementary Table 1). The genetic diversity of these available sources is unknown. Throughout this paper we use the term "accession" to describe our tea plant selections, because the nursery names do not represent true cultivated varieties. We are defining a tea accession as a lot of plants purchased from a single grower under a single name.

Researchers in the United States are establishing living genetic germplasm collections (LeCompte and $\mathrm{Bi}$, personal communication 2018); however, there is little or no knowledge of how these currently available tea plants will perform under disease pressure in U.S. growing conditions. There is also no information available about the $C$. camelliae isolates present in the United States; the only published documentation of $C$. camelliae on U.S.-grown tea is the registration of six herbarium specimens, submitted in 1903 and 1907, in the U.S. National Fungus Collections (Farr and Rossman 2019). Our objectives were to (i) characterize $C$. camelliae isolates collected in north-central Florida, including gene sequence data for key informative regions and phylogenetic relationships to other known tea isolates; (ii) evaluate tea accessions available in the southeastern United States for susceptibility to anthracnose, using a detached leaf assay; and (iii) compare results of in vitro susceptibility testing to observations of disease severity during the 2017 and 2018 growing seasons at the experimental field plot in which the disease symptoms were initially noted.

\section{Materials and Methods}

Pathogen characterization. Seven fungal isolates were obtained between fall 2016 and fall 2018 from symptomatic lesions on Camellia spp. plants in a research plot $(n=2)$, three retail nurseries $(n=4)$, and a botanical garden $(n=1)$ in north-central Florida. Six of the plants were $C$. sinensis, and one was $C$. japonica. The anthracnose lesions on both hosts started at leaf tips, edges, or a site of wounding. Lesions were circular and brown, with a gray or tattered center. Some also had a chlorotic halo around the leading edge, and many showed internal concentric bands of brown and dark brown, with black acervuli visible under a hand lens. For isolations, we surface sterilized symptomatic leaves for $1 \mathrm{~min}$ in $5 \%$ (v/v) commercial bleach ( $7.86 \%$ sodium hypochlorite), rinsed once for $5 \mathrm{~min}$ in sterile deionized water (DI water), plated tissues from leading edges of the lesions on acidified potato dextrose agar (APDA), and then incubated at room temperature $\left(23\right.$ to $\left.25^{\circ} \mathrm{C}\right)$ under a light bank with continuous light for 7 days. One colony type was consistently observed from all camellia samples. We performed microscopy to characterize the fungal morphology for genus confirmation and eventual comparison with the species holotype description (Massee 1899). For DNA extraction, we grew single-spore isolates on APDA, obtained DNA with a cetyltrimethylammonium bromide extraction method (Gardes and Bruns 1993), and then amplified and sequenced the ribosomal internal transcribed spacer (ITS), glutamine synthetase (GS), glyceraldehyde-3-phosphate dehydrogenase (GAPDH), $\beta$-tubulin 2 (TUB2), and mating type protein and Apn2-Mat1-2 intergenic spacer (ApMat) domains using primers reported by Wang et al. (2016). These genes were selected for sequencing because they support a more accurate identification than ITS alone within the genus Colletotrichum (Liu et al. 2015). We sent purified DNA samples to Eurofin (Louisville, KY) and Genewiz (South Plainfield, NJ) for sequencing using the Sanger method, and returned sequences were edited using Geneious 11.1.5 (https://www.geneious.com).

Phylogenetic tree construction. A multigene (ITS, GS, GAPDH, TUB2, and ApMat) phylogeny was constructed using seven sequences generated in this study and 11 previously published sequences from Colletotrichum spp. isolated from tea and ornamental Camellia spp. in China and the United States (Liu et al. 2015). Bayesian analyses were performed on concatenated alignments using MrBayes version 3.2.6 (Huelsenbeck and Ronquist 2001), using nucleotide substitution model GTR with parameters set at chain length of 20 million generations, sampling frequency of 5,000, with the first $10 \%$ of samples being discarded as the burn-in. The remaining parameters were set at program defaults. Maximum likelihood analyses were performed on the same concatenated alignments using PhyML 3.3.20180621 (Guindon et al. 2010) with substitution model HKY85 run for 2,000 bootstraps. All other parameters were set as default.

Field disease severity. Disease severity ratings were recorded at an existing research plot established at the PSREC in Citra, Florida, in March 2016, where anthracnose disease was naturally occurring. This plot originally consisted of eight tea accessions under drip irrigation, with 15 plants per accession in each of three replicate blocks, in a randomized complete block design. Not all accessions that were used in the detached leaf assay occurred in the research field plot, so field observations are limited to the accessions Fairhope, Georgian, Big Leaf, and Small Leaf. We evaluated the plants monthly for anthracnose symptoms. To estimate disease severity, we selected three evenly spaced branches around the central trunk on each plant and scored 10 leaves per branch, beginning at the first mature leaf, for a total of 30 youngest mature leaves per plant. Severity ratings were recorded as number of symptomatic leaves out of 30 leaves per plant. Area under the disease progress curve (AUDPC) was calculated from monthly severity ratings during the 2017 and 2018 growing seasons (April through November). Data were analyzed by ANOVA, and means were compared using Tukey's HSD method in JMP (JMP, Version Windows 7 Ultimate x64, SAS Institute, Cary, NC, 1989 to 2007).

Plant material for detached leaf assay. We used six tea varieties commercially available in the southern United States (Table 1). Plants were grown in a 50/50 pine bark/Fafard number 2 potting mix and were maintained in a greenhouse at temperatures ranging from 10 to $27^{\circ} \mathrm{C}$. Due to a cooling system outage in the greenhouse, the plants experienced an unforeseen heat stress event just prior to the second repetition. Plants were watered four to five times per week as needed and fertilized twice per year with a slow-release fertilizer (Osmocote 14-1414). Greenhouse photosynthetic conditions were $20 \%$ shade with a PPFD value of 870 and $770 \mu \mathrm{mol} /\left(\mathrm{s} \cdot \mathrm{m}^{2}\right)$, taken at noon in July and September, respectively. Plant materials for the three repetitions were collected on the same day as each experiment in May, July, and November.

Table 1. Information on accession names, ploidy, and propagation type, as provided by suppliers

\begin{tabular}{lllll}
\hline Accession & Abbreviation & \multicolumn{1}{c}{ Origin } & Ploidy \\
\hline Small Leaf & MS. SL & Mississippi State University & Diploid & Propagation type \\
Big Leaf & MS. BL & Mississippi State University & Suspected tetraploid & Cuttings from selected seed-grown plants \\
Fairhope & MS. FH & Mississippi State University & Diploid & Cuttings from selected plants \\
Georgian & MS. GEO & Mississippi State University & Diploid & Cuttings (three original parental plants) \\
Black Sea & CF. BS & Camellia Forrest Nursery & Diploid & Seed \\
Korean & CF. KO & Camellia Forrest Nursery & Diploid & Seed \\
\hline
\end{tabular}


Detached leaf assay. We performed detached leaf assays to compare susceptibility across six accessions, using the second and third youngest mature leaves from greenhouse-grown, asymptomatic plants. Leaves were surface sterilized by washing in a 5\% bleach solution (sodium hypochlorite) for $1 \mathrm{~min}$ and then rinsed with sterilized DI water for at least $5 \mathrm{~min}$. During the surface sterilization, we left each leaf attached to a stem segment to reduce phytotoxicity effects of bleach in the leaf vascular tissue. Isolate CaSi 1.1 was selected for inoculations because this isolate originated from the research plot where disease ratings were performed and was a good representation of C. camelliae occurring in north-central Florida (Fig. 1). Cultures of $C$. camelliae used for inoculation were grown on APDA for approximately 2 weeks. Detached leaf assays were performed as described in Yoshida and Takeda (2006), with modifications as follows. Six leaves of each of the six accessions were wounded twice at equal distances from the leaf midrib and the edge of the leaf, at the widest part of the leaf, using a needle probe. The maximum wound area was $4.18 \mathrm{~mm}^{2}$. A conidial suspension was prepared containing $1.1 \times 10^{5}$ spores $/ \mathrm{ml}$ (first repetition), $4.4 \times 10^{4}$ spores $/ \mathrm{ml}$ (second repetition), or $8.7 \times 10^{5}$ spores $/ \mathrm{ml}$ (third repetition), and $25 \mu 1$ was pipetted onto each wound site. Six control leaves were treated with $25 \mu \mathrm{l}$ of sterilized DI water. All leaves were incubated lying flat in an enclosed moist chamber at $22^{\circ} \mathrm{C}$ for $24 \mathrm{~h}$, after which petioles were detached from remaining stem segments and leaves were transferred to Rootcubes (Smithers-Oasis, Kent, $\mathrm{OH}$ ) and arranged in a randomized complete block design. We used Rootcubes to prolong the life of the leaves and reduce physiological stress during the duration of the experiment (Yoshida and Takeda 2006). Leaves in Rootcubes were incubated at $25^{\circ} \mathrm{C}$ and $95 \%$ humidity under a 12-h/12-h light cycle for 15 days. This experiment was repeated three times. We photographed individual leaves 14 days after inoculation and measured lesion area using ImageJ software (W. S. Rasband, ImageJ, U.S. National Institutes of Health, Bethesda, MD, https://imagej.nih. gov/ij/, 1997 to 2016). We reisolated C. camelliae (identified by morphology) from all lesions at the conclusion of each experiment.
Statistical analysis was conducted using the general linear mixed model (PROC GLIMMIX), suitable for unbalanced data, in SAS (version 9.4, SAS Institute). PROC GLIMMIX uses the least-squares method to fit data to a general linear model. Treatment was used as a fixed effect, and replications were used as the random effect. Fixed effects were tested for significance at $\alpha=0.05$, and least squares means were separated using paired $t$ tests.

\section{Results}

Pathogen characterization. Microscopic examination of lesions supported identification of the pathogen as a Colletotrichum species within the $C$. gloeosporioides complex. Under 40x magnification, smooth, dark brown setae were visible inside the acervuli. Conidia were cylindrical, one celled, smooth walled, hyaline, and approximately 14 to $17 \times 3$ to $6 \mu \mathrm{m}(n=25)$ (Supplementary Fig. 1$)$. Our isolates matched the holotype description of $C$. camelliae, providing a tentative identification of this species (Massee 1899). BLAST analysis comparing sequences of five genetic regions informative for species identification within the genus Colletotrichum (ITS, GS, GAPDH, TUB2, and ApMat) with sequences in the NCBI database (NCBI 2019) supported the identification of our isolates as $C$. camelliae. Our phylogenetic tree showed that our north-central Florida isolates were distinctly clustered within the $C$. camelliae clade (Fig. 1). The isolates we collected from nursery- and field-grown tea plants in north-central Florida grouped with C. camelliae isolates from Camellia sinensis in China, whereas the isolate from an ornamental camellia (Camellia japonica) was more closely related to $C$. camelliae isolated from other Camellia spp. in both the United States and China (Fig. 1). The DNA sequences for the isolates used in this study are deposited in GenBank (Supplementary Table 2).

Field disease observations. All four accessions showed incidence of anthracnose in the field. Georgian had the highest disease severity ratings during both the 2017 and 2018 growing seasons (Fig. 2) with AUDPCs that were significantly larger than those for Big Leaf, Fairhope, and Small Leaf $(P<0.02)$. During the 2017 growing season,

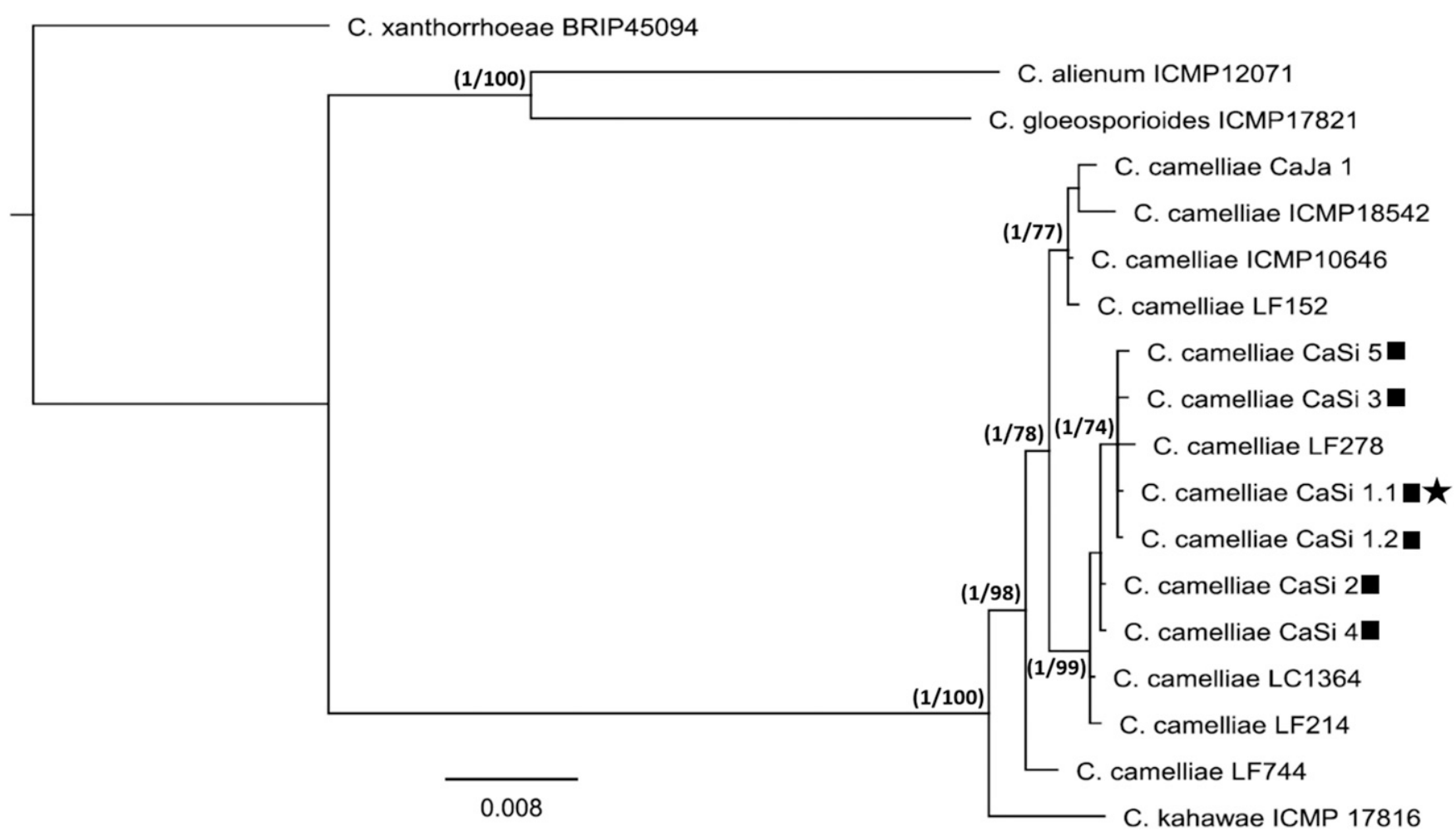

Fig. 1. Seventy percent majority rule consensus tree from maximum likelihood analysis based on five-gene combined dataset (ITS, GAPDH, TUB2, GS, and ApMat) showing phylogenetic affinities of Colletotrichum camelliae isolates found in Florida compared with other known isolates. The maximum likelihood (ML) bootstrap support values (ML > 70 ) and Bayesian posterior probabilities (PP > 0.99) are displayed at the nodes (PP/ML). The tree was rooted to $C$. xanthorrhoeae (BRIP 45094). The scale bar indicates 0.008 expected changes per site. Florida tea isolates are indicated with a square. Isolate used for pathogenicity testing is represented with a star. 
Big Leaf had the smallest AUDPC among the four tested accessions, but this was not significantly different from AUDPCs for Fairhope or Small Leaf. During the 2018 growing season, Small Leaf had the smallest AUDPC among the four tested accessions, but this was not significantly different from AUDPCs for Big Leaf or Fairhope.

Detached leaf assays. All accessions were susceptible to infection by $C$. camelliae, with Fairhope consistently having the largest average lesion size in all three repetitions. Fairhope and Small Leaf had significantly larger lesion sizes than Big Leaf and Black Sea $(P<$ $0.05)$, whereas lesions on Georgian and Korean were not statistically different from either the largest or smallest lesion sizes (Table 2). Control leaves during the first and third experiment of the detached leaf assay produced no lesions; however, one control during the second repetition was contaminated and produced an anthracnose lesion.

\section{Discussion}

Anthracnose caused by C. camelliae is present in the United States and may be problematic for a developing U.S.-based tea industry. In this paper we have provided a characterization of six $C$. camelliae isolates from tea in north-central Florida, including gene sequence data for key informative regions, and evaluated common U.S. tea accessions for susceptibility to anthracnose.

Lesions developed on all six of the tested accessions in the detached leaf assays (Table 2). However, lesion area did differ across the accessions. In all three repetitions of the experiment, Fairhope had the largest lesion size, which suggests that this accession may be the most susceptible to anthracnose disease caused by $C$. camelliae. Of the six accessions tested, Fairhope is one of the most widely planted due to the availability of the planting material. This accession is a derivative of three parental plants grown in a research trial by Lipton at Auburn University (Donnie Barrett, personal communication) and represents some of the oldest available plant material in the United States.

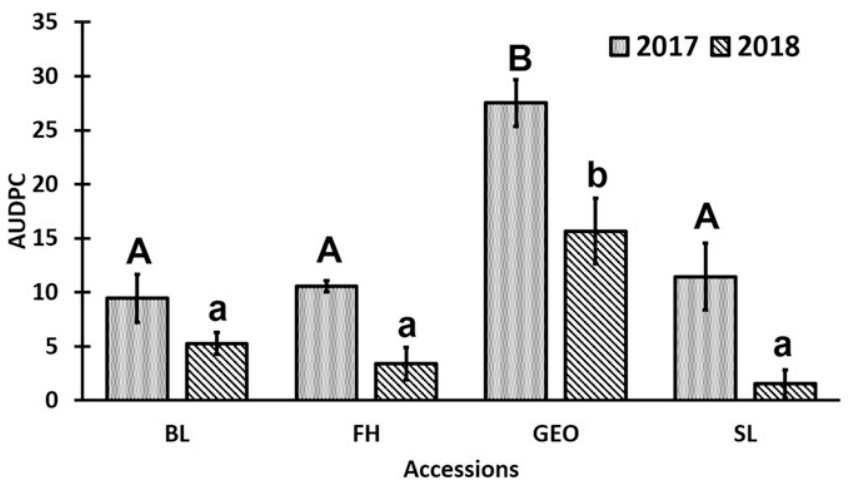

Fig. 2. Area under the disease progress curve (AUDPC) generated from mean severity ratings in the three replicate blocks of each accession $(n=3)$. Accession abbreviations: $\mathrm{FH}=$ Fairhope; GEO = Georgian; $\mathrm{BL}=$ Big Leaf; and $\mathrm{SL}=$ Small Leaf. Means not significantly different $(P<0.02)$ using Tukey's HSD test are marked by the same letters.

Table 2. Anthracnose mean lesion sizes on tea leaves inoculated with Colletotrichum camelliae

\begin{tabular}{lccc}
\hline Accession & Lesion no. & Lesion size $\left(\mathbf{m m}^{\mathbf{2}}\right)^{\mathbf{z}}$ & Standard error \\
\hline Fairhope & $35 / 36$ & $38.05 \mathrm{a}$ & 10.04 \\
Small Leaf & $35 / 36$ & $36.02 \mathrm{a}$ & 9.46 \\
Korean & $35 / 36$ & $23.29 \mathrm{ab}$ & 6.16 \\
Georgian & $34 / 36$ & $22.04 \mathrm{ab}$ & 5.90 \\
Black Sea & $34 / 36$ & $17.41 \mathrm{~b}$ & 4.49 \\
Big Leaf & $29 / 36$ & $12.07 \mathrm{~b}$ & 3.48 \\
\hline
\end{tabular}

y Number of lesions per 36 inoculation points; successful inoculation was defined as having an area larger than the control wound area.

${ }^{\mathrm{z}}$ Data are the mean lesion size of 36 inoculations on 18 leaves and were separated using paired $t$ tests. Means that share the same letter within a column are not significantly different $(P<0.05)$.
We observed variability among the first, second, and third repetitions during the detached leaf assay (Supplementary Table 3), which might be explained by physiological states of the tea plants at time of each experiment. Repeating the experiment at different times of the year better represents the range of physiological states throughout the plant reproductive cycle and under varying environmental conditions but introduces a source of variability into the data. Further study would be needed to elucidate the combination of factors responsible for the variability among our experimental repetitions, but the general lack of significant differences among accessions indicates that all tested accessions in the United States are susceptible to this disease. Relative consistency in ranking of some accessions (e.g., Fairhope with the largest lesion size in two of three repetitions, Georgian consistently in the lower half of the range) may provide some indications of quantitative resistance, which can serve as a foundation for future research.

Field observations indicated that anthracnose will indeed be a significant problem in U.S. tea production, but natural occurrence in the field did not consistently correspond with results from the detached leaf assays. Despite Fairhope and Small Leaf having the largest mean lesion sizes in all repetitions of the detached leaf assay, these accessions had the smallest AUDPCs for disease severity during the 2017 and 2018 growing seasons. In contrast, Georgian had one of the largest AUDPC ratings and one of the smaller mean lesion sizes in the detached leaf assays. Big Leaf had the smallest mean lesion size in the detached leaf assays and had the smallest AUDPC ratings. Inconsistencies between our experimental testing and our field observations might be explained by a whole-plant physiological response to the pathogen. Our detached leaf assays examined how large the lesions developed in a controlled environment. Plants in the field often experience elevated temperatures and other stress events that will likely influence the disease severity. Plants in the field are also able to senesce diseased and damaged leaves. Senesced leaves were not included in disease ratings from our field observations, which introduces a bias toward higher ratings for those accessions that retain diseased leaves longer. The higher humidity conditions during the detached leaf assay may also have resulted in development of larger lesions than would typically occur in the field. This effect could be clarified in future experiments by inoculating whole plants to better match field conditions and observing leaf senescence in addition to disease severity.

The multigene phylogenetic tree showed that our north-central Florida isolates were distinctly clustered in the $C$. camelliae clade (Fig. 1). However, the single isolate we obtained from a Camellia japonica clustered more closely with other isolates from non-tea Camellia species. This may indicate differences in host preference among $C$. camelliae isolates. Future phylogenetic work and pathogenicity assays will include additional ornamental camellia isolates to better understand potential host specificity within this species. Additional phylogenetic analyses will also include isolates from other tea growing regions in Florida and elsewhere in the United States, in order to better characterize pathogen diversity and likely pathways of pathogen movement into and within the United States.

Our results indicate that anthracnose disease on tea should be a focus of research for the development of a U.S.-based tea industry. Tested accessions had varied responses to the disease, both in experimental testing and in our field observations. These results will support future breeding efforts toward developing cultivars for tea production in the southeastern United States. Our future studies are focused on applying the methods developed here to identify anthracnose-tolerant accessions well adapted to Florida growing conditions.

\section{Acknowledgments}

The authors wish to acknowledge Kristen Sandsted for her assistance in collecting data, Dr. Rosanne Healy for her help with microscopy, Dr. Edzard van Staten for statistical consulting, and Nattapol Kraisitudomsook and Brett Lane for assistance with phylogenetic data analysis. We also thank Dr. Erica Goss for providing insightful critical review of this manuscript prior to submission. 


\section{Literature Cited}

Chang, K. 2015. World Tea Production and Trade: Current and Future Development. FAO, Rome, Italy.

Chen, L., and Yamaguchi, S. 2002. Genetic diversity and phylogeny of tea plant (Camellia sinensis) and its related species and varieties in the section Thea genus Camellia determined by randomly amplified polymorphic DNA analysis. J. Hortic. Sci. Biotechnol. 77:729-732.

Chen, Y. J., Qiao, W. J., Zeng, L., Shen, D. H., Liu, Z., Wang, X. S., and Tong, H. R. 2017. Characterization, pathogenicity, and phylogenetic analyses of Colletotrichum species associated with brown blight disease on Camellia sinensis in China. Plant Dis. 101:1022-1028.

Codeminders. 2017. Ocala weather comparison. http://www.codeminders.com/ weather_similarity/.

Farr, D. F., and Rossman, A. Y. 2019. Fungal databases. U.S. National Fungal Collections, ARS, USDA. Retrieved July 15, 2019, from https://nt.arsgrin.gov/fungaldatabases/.

Gardes, M., and Bruns, T. D. 1993. ITS primers with enhanced specificity for basidiomycetes-Application to the identification of mycorrhizae and rusts. Mol. Ecol. 2:113-118.

Guindon, S., Dufayard, J. F., Lefort, V., Anisimova, M., Hordijk, W., and Gascuel, O. 2010. New algorithms and methods to estimate maximum-likelihood phylogenies: Assessing the performance of PhyML 3.0. Syst. Biol. 59:307-321.

Huelsenbeck, J. P., and Ronquist, F. 2001. MRBAYES: Bayesian inference of phylogenetic trees. Bioinformatics 17:754-755.

Liu, F., Weir, B. S., Damm, U., Crous, P. W., Wang, Y., Liu, B., Wang, M., Zhang, M., and Cai, L. 2015. Unravelling Colletotrichum species associated with Camellia: Employing ApMat and GS loci to resolve species in the $C$. gloeosporioides complex. Persoonia 35:63-86.

Massee, G. 1899. Tea and coffee diseases. Pages 89-94 in: Bulletin of Miscellaneous Information, Royal Botanic Gardens. Kew Royal Gardens, Kew, U.K.

NCBI. 2019. NCBI BLAST Library. https://www.ncbi.nlm.nih.gov/blast. Accessed May 2019.

Oniki, M., Hamaya, E., and Ando, Y. 1985. The actual note of resistant strains of the tea anthracnose fungus Gloeosporium theae-sinensis against benzimidazole fungicides in Japan. Chagyo Kenkyu Hokoku (Tea Research Journal) 61:7-11.

Orrock, J. M., Richter, B. S., and Rathinasabapathi, B. 2017. Tea as a novel crop for Florida: Field establishment of eight accessions. Proc. Fla. State Hortic. Soc. 130:35-36.
Pettigrew, J. 2018. World of Tea. 83 Press, Birmingham, AL.

Shepard, C. U. 1899. Tea Culture: The Experiment in South Carolina (Report No. 61). U.S. Department of Agriculture, Washington, D.C. Retrieved April 24, 2018, from https://archive.org/details/teacultureexperi61shep.

Shi, N. N., Du, Y. X., Ruan, H. C., Yang, X. J., Dai, Y. L., Gan, L., and Chen, F. R. 2018. First report of Colletotrichum fructicola causing anthracnose on Camellia sinensis in Guangdong Province, China. Plant Dis. 102: 241-242.

Sreenivasaprasad, S., and Talhinhas, P. 2005. Genotypic and phenotypic diversity in Colletotrichum acutatum, a cosmopolitan pathogen causing anthracnose on a wide range of hosts. Mol. Plant Pathol. 6:361-378.

Tea Association of the U.S.A. 2018. Tea fact sheet 2018-2019. http://www.teausa.com/ 14655/tea-fact-sheet

Teeverband, D. 2018. Global production and exports of tea from 2004 to 2015 (in million metric tons). In: Statista - The Statistics Portal. Retrieved July 19, 2018, from https://www.statista.com/statistics/264183/global-production-and-exportsof-tea-since-2004/.

Vijayan, K., Zhang, W. J., and Tsou, C. H. 2009. Molecular taxonomy of Camellia (Theaceae) inferred from nrITS sequences. Am. J. Bot. 96:1348-1360.

Wang, Y. C., Hao, X. Y., Wang, L., Xiao, B., Wang, X. C., and Yang, Y. J. 2016. Diverse Colletotrichum species cause anthracnose of tea plants (Camellia sinensis (L.) O. Kuntze) in China. Sci. Rep. 6:35287.

Wei, C., Yang, H., Wang, S., Zhao, J., Liu, C., Gao, L., Xia, E., Lu, Y., Tai, Y., She, G., Sun, J., Cao, H., Tong, W., Gao, Q., Li, Y., Deng, W., Jiang, X., Wang, W., Chen, Q., Zhang, S., Li, H., Wu, J., Wang, P., Li, P., Shi, C., Zheng, F., Jian, J., Huang, B., Shan, D., Shi, M., Fang, C., Yue, Y., Li, F., Li, D., Wei, S., Han, B. Jiang, C., Yin, Y., Xia, T., Zhang, Z., Bennetzen, J. L., Zhao, S., and Wan, X. 2018. Draft genome sequence of Camellia sinensis var. sinensis provides insights into the evolution of the tea genome and tea quality. Proc. Natl. Acad. Sci. U.S.A. 115:E4151-E4158.

Weir, B. S., Johnston, P. R., and Damm, U. 2012. The Colletotrichum gloeosporioides species complex. Stud. Mycol. 73:115-180.

Yoshida, K., Ogino, A., Yamada, K., and Sonoda, R. 2010. Induction of disease resistance in tea (Camellia sinensis L.) by plant activators. Jpn. Agric. Res. Q. 44:391-398.

Yoshida, K., and Takeda, Y. 2006. Evaluation of anthracnose resistance among tea genetic resources by wound-inoculation assay. Jpn. Agric. Res. Q. 40:379-386. 